\title{
Observations of the scale-dependent turbulence and evaluation of the flux-gradient relationship for sensible heat for a closed Douglas-fir canopy in very weak wind conditions
}

\author{
D. Vickers and C. K. Thomas \\ Oregon State University, College of Earth, Ocean, and Atmospheric Sciences, Corvallis, Oregon, USA \\ Correspondence to: D. Vickers (vickers@ coas.oregonstate.edu)
}

Received: 17 March 2014 - Published in Atmos. Chem. Phys. Discuss.: 13 May 2014

Revised: 1 August 2014 - Accepted: 8 August 2014 - Published: 16 September 2014

\begin{abstract}
Observations of the scale-dependent turbulent fluxes, variances, and the bulk transfer parameterization for sensible heat above, within, and beneath a tall closed Douglas-fir canopy in very weak winds are examined. The daytime sub-canopy vertical velocity spectra exhibit a double-peak structure with peaks at timescales of $0.8 \mathrm{~s}$ and $51.2 \mathrm{~s}$. A double-peak structure is also observed in the daytime sub-canopy heat flux co-spectra. The daytime momentum flux co-spectra in the upper bole space and in the sub-canopy are characterized by a relatively large crosswind component, likely due to the extremely light and variable winds, such that the definition of a mean wind direction, and subsequent partitioning of the momentum flux into along- and cross-wind components, has little physical meaning. Positive values of both momentum flux components in the sub-canopy contribute to upward transfer of momentum, consistent with the observed sub-canopy secondary wind speed maximum. For the smallest resolved scales in the canopy at nighttime, we find increasing vertical velocity variance with decreasing timescale, consistent with very small eddies possibly generated by wake shedding from the canopy elements that transport momentum, but not heat. Unusually large values of the velocity aspect ratio within the canopy were observed, consistent with enhanced suppression of the horizontal wind components compared to the vertical by the very dense canopy.

The flux-gradient approach for sensible heat flux is found to be valid for the sub-canopy and above-canopy layers when considered separately in spite of the very small fluxes on the order of a few $\mathrm{W} \mathrm{m}^{-2}$ in the sub-canopy. However, singlesource approaches that ignore the canopy fail because they make the heat flux appear to be counter-gradient when in
\end{abstract}

fact it is aligned with the local temperature gradient in both the sub-canopy and above-canopy layers. While sub-canopy Stanton numbers agreed well with values typically reported in the literature, our estimates for the above-canopy Stanton number were much larger, which likely leads to underestimated modeled sensible heat fluxes above dark warm closed canopies.

\section{Introduction}

Observational studies are important for improving our basic understanding of the turbulence mixing and turbulence transport for different forest canopy architectures in varying conditions (e.g., Baldocchi and Meyers, 1988; Meyers and Baldocchi, 1991; Raupach, 1994; Raupach et al., 1996; Vickers and Thomas, 2013, and references therein). Such studies are also important for more practical problems including mixing of scalars in the sub-canopy, decoupling of the sub-canopy (e.g., Staebler and Fitzjarrald, 2005; Vickers et al., 2012; Thomas et al., 2013; Vickers and Thomas, 2013), air-surface exchange processes (e.g., Goulden et al., 1996; Thomas et al., 2013), and parameterizations of the turbulent fluxes in terms of model-resolved bulk quantities, such as flux-gradient methods and Monin-Obukhov similarity theory.

The observations in this study are of special interest because they are characterized by a very dense canopy and very weak winds. The sub-canopy has a reversed heat flux regime, where the heat flux is upward at night and downward during the day. The daytime momentum flux is typically upwards in 
Table 1. Daytime averages of the wind speed $\left(U, \mathrm{~ms}^{-1}\right)$, vertical velocity variance $\left(<w^{\prime} w^{\prime}>, \mathrm{m}^{2} \mathrm{~s}^{-2}\right)$, kinematic heat flux $\left(<w^{\prime} \theta^{\prime}>, \mathrm{Km} \mathrm{s}^{-1}\right)$, along-wind momentum flux component $(<$ $\left.w^{\prime} u^{\prime}>, \mathrm{m}^{2} \mathrm{~s}^{-2}\right)$, and the cross-wind momentum flux component $\left(<w^{\prime} v^{\prime}>, \mathrm{m}^{2} \mathrm{~s}^{-2}\right.$ ) at $38 \mathrm{~m}$ a.g.l. (above-canopy), $16 \mathrm{~m}$ (lower crown space), and $4 \mathrm{~m}$ (sub-canopy).

\begin{tabular}{rrrrrr}
\hline$z(\mathrm{~m})$ & $U$ & $\left\langle w^{\prime} w^{\prime}\right\rangle$ & $\left\langle w^{\prime} \theta^{\prime}\right\rangle$ & $\left\langle w^{\prime} u^{\prime}\right\rangle$ & $\left\langle w^{\prime} v^{\prime}\right\rangle$ \\
\hline 38 & 2.3 & 0.59 & 0.20 & -0.36 & 0.0030 \\
16 & 0.19 & 0.13 & 0.0076 & -0.0064 & 0.0081 \\
4 & 0.56 & 0.014 & -0.0024 & 0.0043 & 0.0077 \\
\hline
\end{tabular}

the sub-canopy associated with a decrease in the mean wind speed with increasing height between the sub-canopy layer and the within-canopy layer. Analysis of such weak turbulence sub-canopy conditions coupled with the reversed heat flux regime is often avoided in the literature.

In this study we analyze observations of the scaledependent turbulent fluxes and variances above, within, and beneath a tall closed Douglas-fir canopy. In addition, we evaluate the standard flux-gradient approach for sensible heat to address the question of whether or not standard fluxgradient methods are appropriate for the sub-canopy layer, where the mean wind speed is very weak and decreases with height, and the primary source of heating and cooling is located at the top of the layer. Such evaluations are relevant to the ecosystem modeling community as their models typically use some form of the flux-gradient relationship and may or may not resolve the different layers separately.

\section{Materials and methods}

\subsection{Site description}

The data analyzed here were collected in a 33-year-old Douglas-fir forest located in the coast range of western Oregon, USA (AmeriFlux site US-Fir, $44.646^{\circ} \mathrm{N}$ latitude, $123.551^{\circ} \mathrm{W}$ longitude, $310 \mathrm{~m}$ elevation) during the period 5 May through 24 October 2007 (Thomas et al., 2008; Thomas, 2011). The vertical structure of the vegetation canopy consists of a sparse understory composed mainly of Salal (Gaultheria shallon) with a maximum plant height of $0.8 \mathrm{~m}$ above ground level (a.g.l.) and the main Douglasfir (Pseudotsuga menziesii) crown space extending from 15 to $26 \mathrm{~m}$ a.g.l. The site is surrounded by moderately sloped terrain with a relatively flat saddle located approximately $600 \mathrm{~m}$ to the northeast of the tower. The canopy is very dense with a plant area index (PAI) of $9.4 \mathrm{~m}^{2} \mathrm{~m}^{-2}$ optically measured in 2004 (Model LAI2000, Licor, Lincoln, NE, USA). The winds and turbulence are very weak (Tables 1 and 2 and Fig. 1). The persistent weak wind above the canopy is thought to be due to topographic sheltering by the coast range with westerly winds.
Table 2. Same as Table 1 but for nighttime averages.

\begin{tabular}{rrrrrr}
\hline$z(\mathrm{~m})$ & $U$ & $\left\langle w^{\prime} w^{\prime}\right\rangle$ & $\left\langle w^{\prime} T^{\prime}\right\rangle$ & $\left\langle w^{\prime} u^{\prime}\right\rangle$ & $\left\langle w^{\prime} v^{\prime}\right\rangle$ \\
\hline 38 & 0.94 & 0.085 & -0.0049 & -0.046 & -0.0067 \\
16 & 0.14 & 0.020 & 0.0074 & -0.00034 & 0.0021 \\
4 & 0.29 & 0.0054 & 0.0015 & 0.00023 & -0.00029 \\
\hline
\end{tabular}
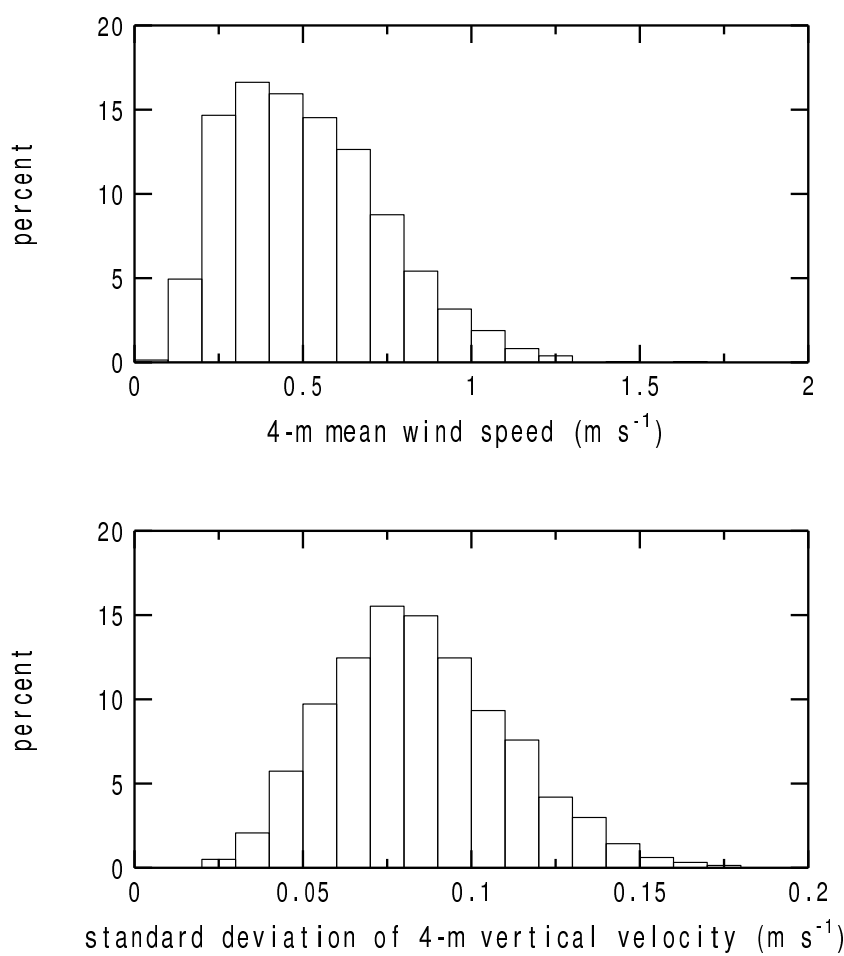

Figure 1. The frequency distribution of the sub-canopy mean wind speed (top) and the standard deviation of vertical velocity (bottom).

\subsection{Instrumentation}

Eddy-covariance measurements of the fast response wind components and temperature were collected using threedimensional sonic anemometers (model CSAT3, Campbell Scientific Inc., Logan, UT, USA) during the summer dry period from 5 May through 24 October 2007. The analysis uses $20 \mathrm{~Hz}$ time series data collected at three levels: $12 \mathrm{~m}$ above the canopy at $38 \mathrm{ma.g.1} .(z / h=1.5)$, at $16 \mathrm{ma.g} .1$. at the transition from the clear bole space of the sub-canopy to the main crown space, and in the open sub-canopy at $4 \mathrm{~m}$ a.g.l. Slow response air temperature was measured using aspirated and shielded sensors (PRT 1000) at 4, 16, and $38 \mathrm{~m}$ a.g.l., and soil temperature was measured with a thermistor $0.02 \mathrm{~m}$ beneath the surface.

The surface brightness temperature of the top of the canopy layer was calculated from the downward-facing longwave pyrgeometer (CNR1, Kipp and Zonen) at $37 \mathrm{~m}$ a.g.l. using an emissivity of the foliage of 0.99 . This value of the emissivity was found to minimize the number of 
counter-gradient heat fluxes for the flux measured at $38 \mathrm{~m}$ and the temperature gradient between the top of the canopy and $38 \mathrm{~m}$. This approach is justified by our high confidence that the $38 \mathrm{~m}$ heat flux should be aligned with the mean temperature gradient above the canopy at $z / h=1.5$.

\subsection{Analysis}

Fluxes and variances are computed using block-averaging where variables are decomposed into a mean part and a turbulent part as

$\phi=\bar{\phi}+\phi^{\prime}$

where the overbar denotes a suitable time average (the perturbation timescale $\tau$ ) and $\phi$ represents the $20 \mathrm{~Hz}$ wind components or temperature. Unlike running means and bandpass filters, block averaging satisfies Reynolds averaging. The time-averaged fluxes and variances are then computed as the average of the instantaneous products of perturbations over some chosen flux-averaging timescale $\lambda$. For example, the vertical turbulent flux of $\phi$ is $\left\langle w^{\prime} \phi^{\prime}\right\rangle$, where the angle brackets indicate averaging over the flux-averaging timescale.

Increasing the perturbation timescale allows larger scale motions to be included in the calculated flux, while a decrease excludes larger scale motions. Increasing the fluxaveraging timescale reduces the random sampling error, but may in turn introduce additional non-stationarity (Vickers et al., 2009). Our primary calculations use $\tau=10 \mathrm{~min}$ and $\lambda=30 \mathrm{~min}$; however, the sensitivity of our results for the Stanton number to the choices of $\tau$ and $\lambda$ is explored below.

Due to the small observed tilt angles and the general uncertainty in the justification for applying a tilt correction (or coordinate rotation), especially for very weak wind sub-canopy data, we did not make any corrections to the fast response wind components to account for a possible tilt in the sonic anemometers from true vertical. The average tilt angle calculated (but not used for any rotations) for the most frequent mean wind direction of west-northwest is less than $1^{\circ}$ in the sub-canopy and $3^{\circ}$ above the canopy. It is not clear whether a non-zero angle indicates real time-averaged vertical motion or a tilted sensor.

Multiresolution decomposition (Howell and Mahrt, 1997; Vickers and Mahrt, 2003) is used to compute the scale dependences of the vertical velocity variance, the heat flux, the momentum flux components, and the velocity aspect ratio. Multiresolution analysis applied to time series decomposes the record into simple unweighted averages on dyadic timescales and represents the simplest possible orthogonal decomposition. Unlike Fourier analysis, multiresolution decomposition satisfies Reynolds averaging at all scales and, as a local transform, it does not assume periodicity of the signals.
The isotropy of the turbulence is examined using the velocity aspect ratio (Vickers and Mahrt, 2006) computed as

$\operatorname{VAR}=\frac{2^{1 / 2} \sigma_{w}}{\left(\sigma_{u}^{2}+\sigma_{v}^{2}\right)^{1 / 2}}$

where $\sigma$ denotes the standard deviation (e.g., $\sigma_{w}={\overline{w^{\prime} w^{\prime}}}^{1 / 2}$ ). In the case where $\sigma_{u}=\sigma_{v}=\sigma_{w}$, VAR (velocity aspect ratio) is unity and the turbulence is isotropic. Small values of VAR indicate mostly two-dimensional motions that are likely nonturbulent.

A normalized turbulence intensity is evaluated as $\sigma_{w} U^{-1}$, where $\sigma_{w}$ is the standard deviation of the vertical velocity and $U$ is the mean wind speed. Large values of the normalized turbulence intensity could indicate that sources of turbulence other than local shear generation are important.

\subsection{Bulk fluxes}

The bulk aerodynamic relationship for estimating surface fluxes is applied in almost all numerical models (e.g., the Community Land Model; Oleson et al., 2010) either directly or indirectly in combination with other approaches. The bulk formulation for the heat flux is typically written as

$H=\rho c_{p}<w^{\prime} \theta^{\prime}>=\rho c_{p} C_{H} U\left(\theta_{o}-\theta\right)$,

where $H$ is the sensible heat flux, $\rho$ is the air density, $c_{p}$ is the specific heat at constant pressure, $\left\langle w^{\prime} \theta^{\prime}\right\rangle$ is the kinematic heat flux, $C_{H}$ is the Stanton number at height $z$ and is the exchange coefficient for sensible heat, $U$ is the mean wind speed at height $z, \theta_{o}$ is the aerodynamic potential temperature, and $\theta$ is the potential temperature of the air at height $z$. The Community Land Model uses the aerodynamic resistance $r_{H}$, which is equal to the inverse of the product of the Stanton number and the mean wind speed, i.e., $r_{H}=\left(C_{H} U\right)^{-1}$.

Using Monin-Obukhov similarity theory, the aerodynamic potential temperature $\theta_{o}$ is defined by vertically integrating the non-dimensional potential temperature gradient from some level in the surface layer down to the roughness length for heat. However, since Monin-Obukhov similarity theory is commonly assumed to be invalid for the roughness sublayer between the surface and the surface layer (e.g., Raupach, 1994), the computed aerodynamic temperature will be different from observed quantities, and therefore, rigorous evaluation of similarity theory is problematic (Mahrt and Vickers, 2004). Our estimates of the sub-canopy $C_{H}$ are made possible by substituting a measured temperature for the aerodynamic potential temperature in Eq. (3). In our case, we use the $2 \mathrm{~cm}$ soil temperature when evaluating the fluxgradient relationship in the sub-canopy and the radiative temperature of the top of the canopy when evaluating the fluxgradient method above the canopy. 

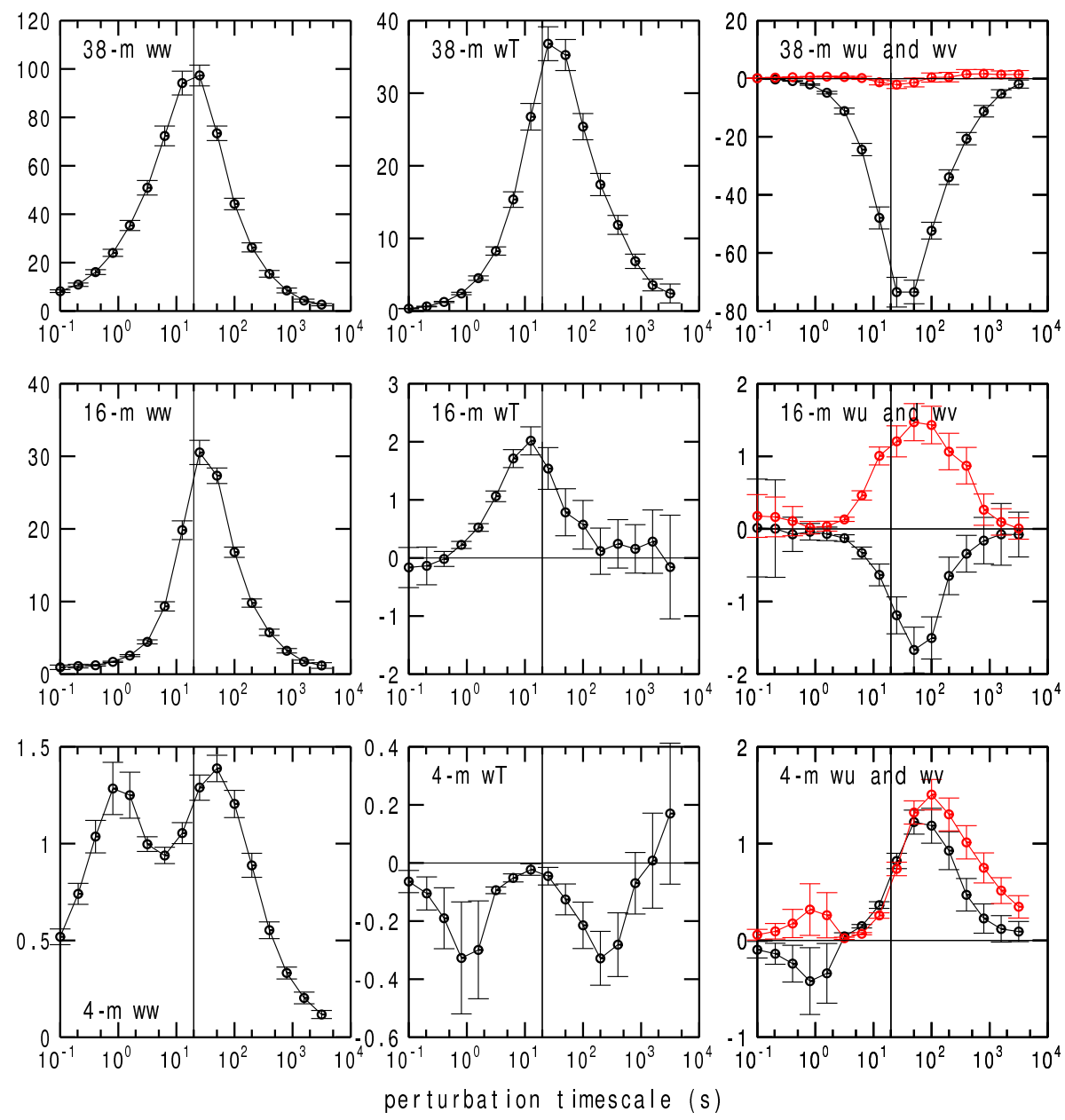

Figure 2. Composites of three levels of daytime vertical velocity spectra ww $\left(\mathrm{m}^{2} \mathrm{~s}^{-2}\right.$, left column), kinematic heat flux co-spectra wT ( $\mathrm{K} \mathrm{m} \mathrm{s}^{-1}$, middle column), and the along- and cross-wind (red) components of the momentum flux (wu and wv) ( $\mathrm{m}^{2} \mathrm{~s}^{-2}$, right column). All quantities have been multiplied by 1000 . The vertical line in each panel denotes $\tau=20 \mathrm{~s}$. The error bars denote the $99 \%$ confidence limit about the mean.

\section{Results}

\subsection{Turbulence structure}

The composite scale-dependent vertical velocity variance, heat flux, and momentum flux components above the canopy at $38 \mathrm{~m}$ a.g.l., at the top of the sub-canopy bole space at $16 \mathrm{~m}$, and in the open sub-canopy at $4 \mathrm{~m}$ during the day and night are shown in Figs. 2 and 3, respectively. To avoid contamination of daytime and nighttime comparisons, the morning and evening transition periods have been excluded from these composites. The daytime heat flux is upward at 16 and $38 \mathrm{~m}$ and downward at $4 \mathrm{~m}$ because the primary daytime radiative heating occurs in the tree crown where the leaf area density is greatest, and not at the ground surface. At night, the $16 \mathrm{~m}$ heat flux remains upward while the $38 \mathrm{~m}$ and $4 \mathrm{~m}$ level heat fluxes switch signs due to strong radiative cooling of the canopy.
Comparing the location of the spectral peaks for, e.g., the vertical velocity variance across levels, shows that the ratio of sub-canopy to above-canopy timescales exceeds two, while the ratio of sub-canopy to upper-boundary of the bole space equals unity for the nighttime and exceeds two for the daytime data. This can be explained by the closed, dense canopy. As evidenced by the direction and magnitude of the heat fluxes, the flow and transport at the 38 and $16 \mathrm{~m}$ levels communicate actively during the day indicating a coupled state, while the $4 \mathrm{~m}$ level is buoyantly decoupled. At night, the 16 and $4 \mathrm{~m}$ levels are closely coupled, while the significant above-canopy stratification decouples observations further aloft at $38 \mathrm{~m}$.

In addition to a shift toward increased peak timescale with decreasing proximity to the ground, we also call attention to the much broader spectral peaks for sub-canopy fluxes and vertical velocity variance compared to the more narrowly defined above-canopy peaks. The former may indicate 

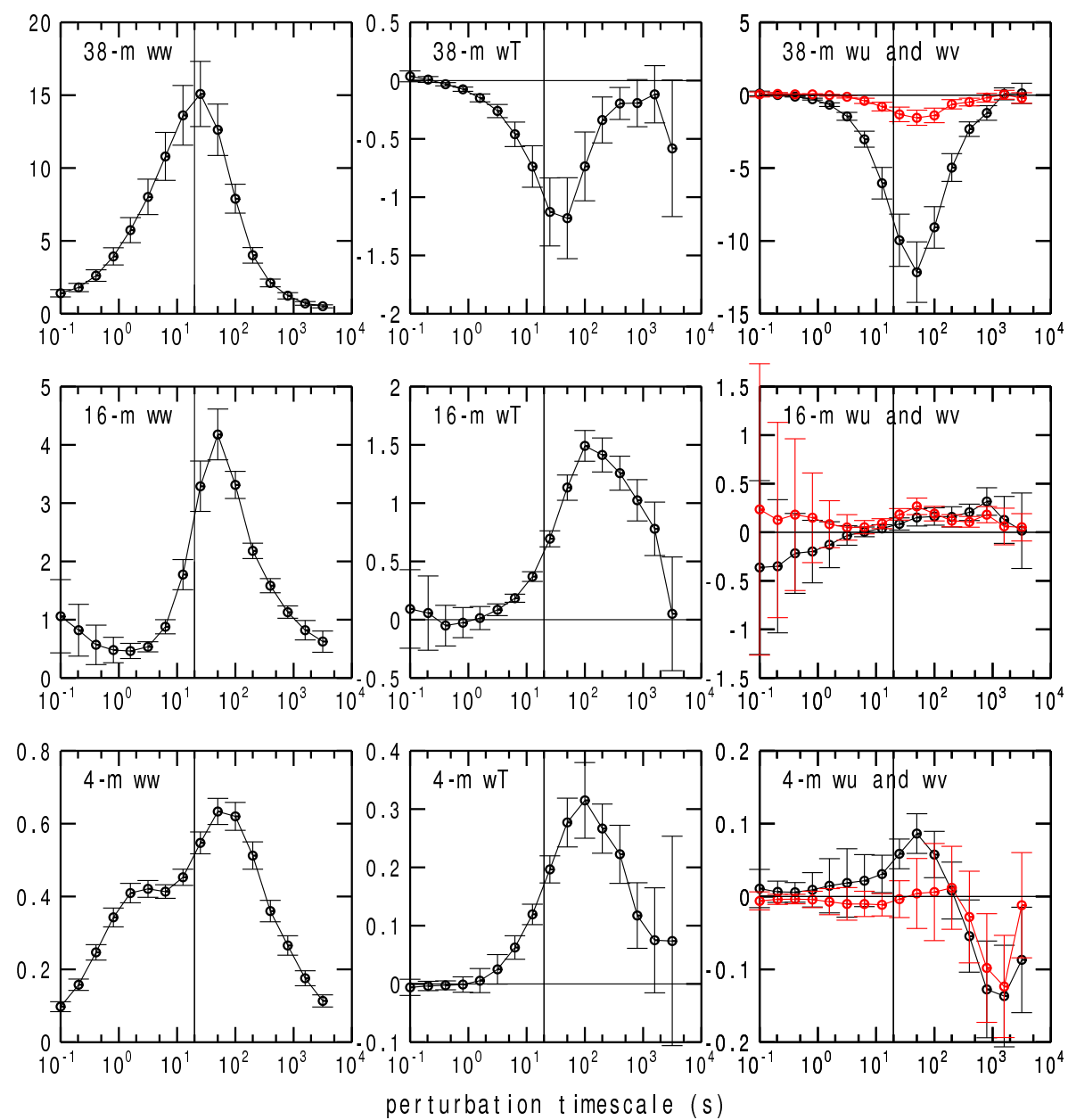

Figure 3. Same as Fig. 2 but for nighttime.

a wide variety of generating mechanisms, while the latter point to buoyancy as the most important mechanism driving the above-canopy turbulence.

The spectra in Figs. 2 and 3 are ensemble-averaged over more than 8200 individual 30 min spectra, and the error bars indicate that the peak in the nighttime momentum flux at timescales of 1000 to $2000 \mathrm{~s}$ is statistically significant. One would expect the momentum transport to be negative, i.e., directed toward the ground, but it is surprising that the largest contribution to the flux is found at such large timescales. We currently do not have a sound physical explanation for the occurrence of these larger scale motions, but note that nonturbulent sub-mesoscale motions are largely responsible for the sub-canopy flow and transport of heat at this site as found in Thomas (2011).

A peculiar double-peak structure in the sub-canopy vertical velocity spectra is found in about two-thirds of all cases during the day, but is absent in almost all nighttime data. The double peaks are found for all wind directions, giving confidence that the result is likely not due to a measurement or flow distortion problem. The first peak is found at $0.8 \mathrm{~s}$, while the timescale of the second peak is $51.2 \mathrm{~s}$ (Fig. 2). The length scale associated with a timescale of $0.8 \mathrm{~s}$ is only about $0.4 \mathrm{~m}$, using a sub-canopy mean wind speed of $0.5 \mathrm{~m} \mathrm{~s}^{-1}$ in combination with Taylor's hypothesis, which may not be reliable in these very weak wind and weak turbulence conditions. Although we have no direct measurements to confirm this, the peak in the vertical velocity spectra at $0.8 \mathrm{~s}$ may be associated with very small eddies generated by wake shedding at the lower edge of the canopy layer (e.g., Meyers and Baldocchi, 1991; Brunet et al., 1994; Dupont et al., 2012). These very small eddies apparently transport momentum but not heat. The lack of a well-defined double-peak structure in the vertical velocity spectra at night may be related to the weaker wind speeds at night (Tables 1 and 2) and subsequently less wake turbulence.

The formation of a double peak in the vertical velocity spectra may also be related to canopy density and could be site specific. The canopy studied here is remarkable for its large plant area index of 9.4. Our previous study (Vickers and Thomas, 2013) looked at a tall open-canopy ponderosa pine site with a plant area index of 2.8. No double peak in 
the vertical velocity spectra was detected in the sub-canopy of the tall open-canopy site

A double-peak structure is also observed in the daytime sub-canopy heat flux co-spectra (Fig. 2), where a local minimum in the negative heat flux is found for the range of timescales from 6.4 to $25.6 \mathrm{~s}$. The heat fluxes at 16 and $4 \mathrm{~m}$ are opposite in direction, but the magnitude of the $4 \mathrm{~m}$ heat flux is only approximately 15 to $25 \%$ of that further aloft. We propose that the heat flux contribution for the timescales dominating the $16 \mathrm{~m}$-flux cancel out near the ground as colder air is moved upward and mixes with the relatively cold, but still warmer air moving downward from the $16 \mathrm{~m}$ level. One would thus expect this canceling effect to create a gap in the $4 \mathrm{~m}$ heat flux co-spectra at timescales around $15 \mathrm{~s}$, as evidenced in Fig. 2, bottom panel. Thus, the co-spectral gap should not be interpreted as evidence that motions on these scales do not exist. We argue that the double peaks are the result of the canceling contribution of opposing fluxes creating a gap in an otherwise continuous cospectrum, and thus must not be interpreted as evidence for physical processes generating motions with two distinctly different timescales of 1 and $200 \mathrm{~s}$. The minimum in the subcanopy vertical velocity variance coincides with timescales around $15 \mathrm{~s}$, and its existence is consistent with our interpretation since the mixing of air and cancelation of opposing fluxes near the ground would lead to suppressed vertical motions due to buoyancy effects.

The daytime momentum flux co-spectra at 16 and $4 \mathrm{~m}$ are characterized by a relatively large cross-wind component (Fig. 2). This is likely due to the extremely light and variable sub-canopy winds, such that the definition of a mean wind direction and subsequent partitioning of the momentum flux into cross- and along-wind components has little physical meaning. Above the canopy at $38 \mathrm{~m}$, where the mean wind speeds are still weak but are much larger than in the subcanopy (Tables 1 and 2), our results agree with those typically found in the literature indicating that the cross-wind component of the momentum flux is typically small compared to the along-wind component. Positive values of both momentum flux components in the sub-canopy at timescales exceeding about $10 \mathrm{~s}$ (Fig. 2) denote a net upward transfer of momentum at these scales and are consistent with the observed decrease in the mean wind speed with height from $4 \mathrm{~m}$ to $16 \mathrm{~m}$.

At the $16 \mathrm{~m}$ level at night, we find relatively large momentum fluxes and vertical velocity variance at the smallest resolved timescales (Fig. 3). However, carefully comparing the day- and nighttime ensemble-averaged momentum flux cospectra shows that these fluxes occur during both day and night with about the same magnitude and sign. Because the scaling of the ordinates is different for the day and night, it is difficult to see the contribution from the smallest motions. The error bars in Fig. 2 and 3 indicate that this contribution at the smallest scales is highly variable in both magnitude

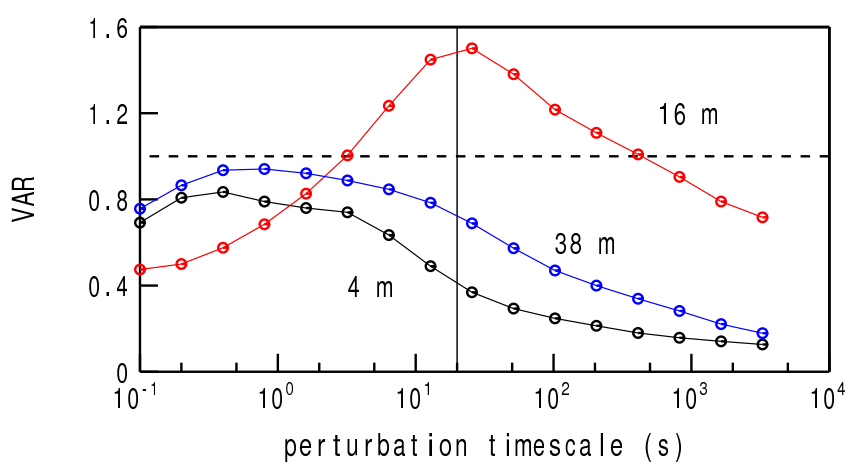

Figure 4. Three levels of the scale dependence of the velocity aspect ratio, VAR. The vertical line denotes $\tau=20 \mathrm{~s}$. The horizontal dashed line represents unity, where the turbulence is isotropic.

and sign, and thus their behavior in the ensemble-averaged spectra should not be over-interpreted.

\subsection{Velocity aspect ratio}

The velocity aspect ratio (Eq. 2) is smaller in the sub-canopy compared to above the canopy at all timescales (Fig. 4), indicating that the sub-canopy motions are more anisotropic (more horizontal) compared to those above the canopy, consistent with enhanced suppression of vertical velocity perturbations closer to the ground. Both above and below the canopy, VAR is maximum at timescales of 0.4 to $0.8 \mathrm{~s}$, and decreases with increasing timescale. The scale dependences of the above-canopy and sub-canopy estimates of VAR are similar to those observed at a tall open-canopy pine forest site (Vickers and Thomas, 2013). The decrease in VAR with decreasing timescale for timescales shorter than $0.4 \mathrm{~s}$ is likely a shortcoming of the instrumentation, possibly due to a preferred path-length-averaging in the vertical direction caused by the sensor geometry of the sonic anemometers.

The unusual scale dependence of VAR at $16 \mathrm{~m}$ suggests that the canopy inhibits horizontal fluctuations more than vertical ones, leading to large values of VAR that can even exceed unity in the long-term average (Fig. 4). At this site, the composite VAR reaches a local maximum of 1.5 at a timescale of $25.6 \mathrm{~s}$. This enhanced suppression of the horizontal wind fluctuations compared to the vertical at a height where the tree crown is densest was qualitatively confirmed by visualizing the canopy flow using episodic releases of artificially generated buoyantly neutral fog. The fog showed strong and sudden vertical motions and enhanced diffusion, while horizontal dispersion and transport were very weak.

While we are not aware of similar observations reported in the literature, the suppression of horizontal over vertical motions can be explained when recalling the physical canopy architecture of the Douglas-fir trees. In the horizontal direction, the overlapping branches including their needles form a large, uniform face and create a large flow resistance when 


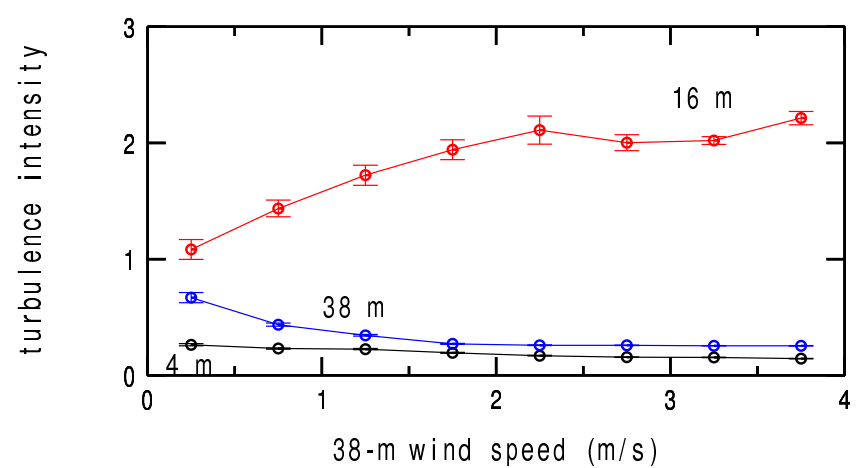

Figure 5. The normalized turbulence intensity at three levels as a function of the wind speed above the canopy. Error bars denote \pm 1 standard error.

averaged over spatial scales exceeding that of a single tree. In the vertical direction, gaps in-between individual trees that are visible from the top of the tower create narrow passages that do not impede the vertical motions. The combined effect of the differences in canopy architecture leads to a greater suppression of horizontal in comparison to vertical motions, thus relatively enhancing the velocity aspect ratio shown in Fig. 4. These results may be site specific.

\subsection{Normalized turbulence intensity}

The normalized turbulence intensity $\left(\sigma_{w} U^{-1}\right)$ at $16 \mathrm{~m}$ is much larger than either above or below the canopy, increases from about 1 to 2 as the $38 \mathrm{~m}$ wind speed increases from 0 to $4 \mathrm{~m} \mathrm{~s}^{-1}$, and levels off for wind speeds exceeding $2 \mathrm{~m} \mathrm{~s}^{-1}$ (Fig. 5). Moving from the upper clear bole space at $16 \mathrm{~m}$ to above the canopy at $38 \mathrm{~m}$, the mean wind speed increases by a factor of 12 while $\sigma_{w}$ increases by only a factor of 2 . That is, the influence of the canopy on the flow is to strongly reduce the mean horizontal wind speed while only weakly reducing the vertical velocity fluctuations. The relative lack of suppression of the vertical velocity perturbations at $16 \mathrm{~m}$ is consistent with the finding above, where the canopy elements act to suppress the horizontal fluctuations more than the vertical ones leading to large VAR at $16 \mathrm{~m}$. We are not aware of any reported values of the turbulence intensity that exceed those observed here (Fig. 5).

\subsection{Flux-gradient relationship in the sub-canopy}

The composite diurnal cycle of the sub-canopy sensible heat flux over the entire experimental period ranges from about $1 \mathrm{~W} \mathrm{~m}^{-2}$ at night to $-3 \mathrm{~W} \mathrm{~m}^{-2}$ during the day (Fig. 6). The fluxes are very small as a result of the extremely weak turbulence due to the combination of weak winds above the canopy and a very dense canopy (Fig. 1). Despite the very weak turbulence and very small fluxes, a coherent temporal pattern is observed where the heat flux is upward at night because the ground surface is warmer than the canopy due to
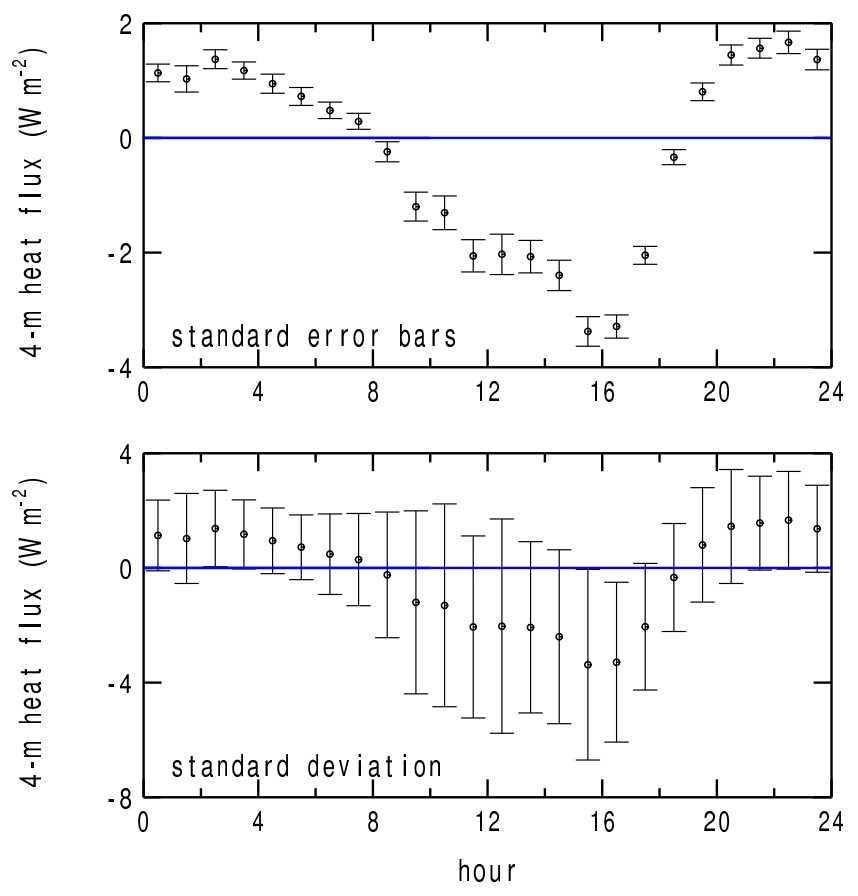

Figure 6. The observed diurnal cycle of the sub-canopy sensible heat flux with standard error bars (top) and \pm 1 standard deviation (bottom), where the uncertainty is due to the day-to-day variability in the heat flux for a given hour of the day over the entire 5-month period.

strong radiational cooling of the tree crowns, and is downward during the day, when the tree crowns are warmer than the ground surface. This diurnal cycle of the heat flux is opposite to the common textbook case where the heat flux is upward during the day and downward at night. It is rather remarkable that a consistent sub-canopy heat flux pattern emerges despite the instrumental challenges of measuring such small heat fluxes characterized by large relative sampling errors associated with the shortcomings of computing eddy-covariance fluxes in very weak turbulence (Mahrt, 2010).

The relationship between the $4 \mathrm{~m}$ heat flux and the product of the mean wind speed and the temperature difference is shown in the bottom panels of Figs. 7 and 9. The slope of the linear regression line is an estimate of the Stanton number $\left(C_{H}\right)$. The estimate for $C_{H}$ of $1.1 \pm 0.04 \times 10^{-3}$ with $r^{2}=$ 0.32 is within the range of typical values reported for $C_{H}$ in the literature ranging from 1 to $5 \times 10^{-3}$ (Stull, 1990). The large relative random sampling errors associated with the small fluxes contribute to the scatter.

We could not find any other variable that explained significant additional variance in the $4 \mathrm{~m}$ heat flux, including soil temperature, soil moisture content, air temperature, wind speed or direction above or below the canopy, moisture flux, momentum flux, or the variances of vertical velocity, temperature, or moisture. That is, the formulation in Eq. (3), 

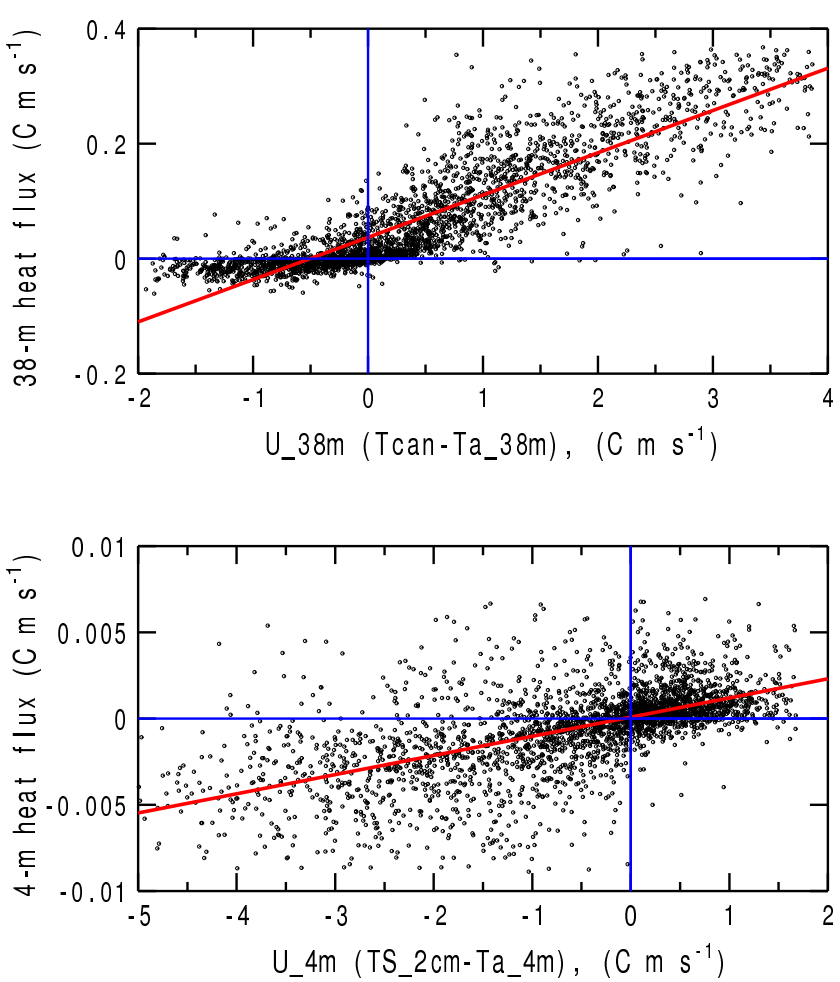

Figure 7. Scatter plot of the $30 \mathrm{~min}$ average sub-canopy kinematic heat flux (lower panel) as a function of the product of the mean wind speed and the temperature difference. The slope of the linear regression line (red) is an estimate of the sub-canopy Stanton number $\left(\mathrm{C}_{H}\right)$. The estimate for the sub-canopy $\mathrm{C}_{H}$ using this approach is $1.1 \pm 0.04 \times 10^{-3}$, using a $90 \%$ confidence interval for the slope, and the regression explains $32 \%$ of the variance. Above the canopy at $38 \mathrm{~m}$ (upper panel), the estimate of the Stanton number is $73.5 \pm$ $1.3 \times 10^{-3}$, with $77 \%$ of the variance explained.

assuming the exchange coefficient is known, appears to be the best formulation of the heat flux, thus confirming the bulk flux-gradient approach for the sub-canopy.

In addition to general agreement with previously reported $C_{H}$ estimates, it is encouraging that the intercept of the regression equals zero for all practical purposes (bottom panels of Figs. 7 and 9), which indicates that, on average and despite the large scatter, the sub-canopy heat fluxes are aligned with the direction of the local temperature gradient as required by the flux-gradient relationship. The zero intercept was found using the $2 \mathrm{~cm}$ soil temperature and the $4 \mathrm{~m}$ shielded and aspirated air temperature to calculate the temperature difference. The zero intercept suggests that the $2 \mathrm{~cm}$ soil temperature measurement is representative of the average ground surface (skin) temperature in the sub-canopy eddy-covariance flux footprint.

The dependence of the strength of the regression relationship (or the fraction of variance explained $\left(r^{2}\right)$ ) and the slope $\left(C_{H}\right)$ on the flux perturbation timescale $(\tau)$ and the fluxaveraging timescale $(\lambda)$ is briefly discussed here. Neither $r^{2}$

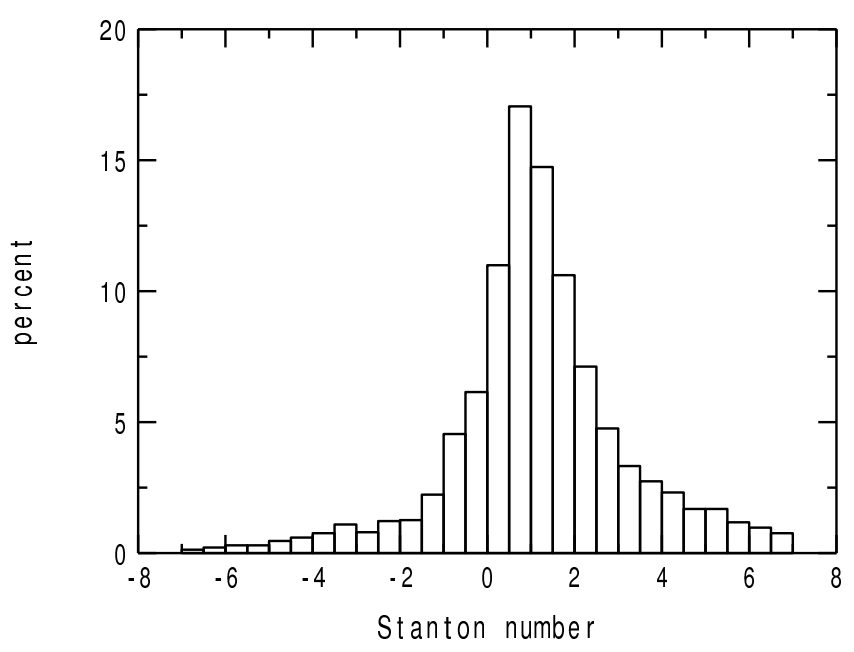

Figure 8. The frequency distribution of the sub-canopy Stanton number (multiplied by 1000) where each 30 min estimate is computed as the heat flux divided by the product of the mean wind speed and the temperature difference. This approach for estimating the Stanton number yields a mean value of $1.1 \times 10^{-3}$ and a standard deviation of $2.05 \times 10^{-3}$.
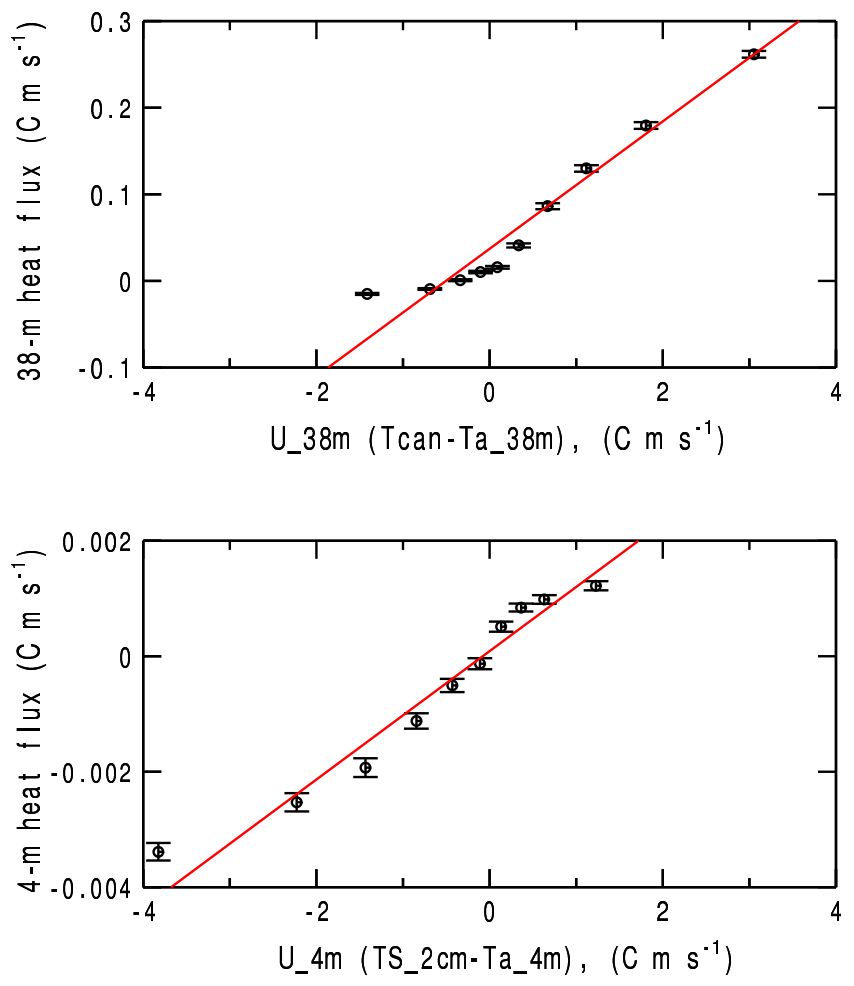

Figure 9. The kinematic heat flux as a function of the product of the mean wind speed and the temperature difference at $38 \mathrm{~m}$ (top panel) and at $4 \mathrm{~m}$ (bottom). The slopes of the linear regression lines (red) are estimates of the Stanton number: $73.5 \pm 1.3 \times 10^{-3}$ at $38 \mathrm{~m}$ and $1.1 \pm 0.04 \times 10^{-3}$ at $4 \mathrm{~m}$. Each of the ten class averages contains an equal number (282) of $30 \mathrm{~min}$ samples. Error bars denote \pm 1 standard error. 
or $C_{H}$ are strongly sensitive to $\lambda$. The variance explained by the regression $\left(r^{2}\right)$ increases slightly with increasing $\lambda$ due to a decrease in the random sampling error. The change in $C_{H}$ with flux-averaging timescale is small, decreasing only $6 \%$ for a change in $\lambda$ from $30 \mathrm{~min}$ to $2 \mathrm{~h}$. The $r^{2}$ decreases slightly with increasing $\tau$ because an increasing perturbation timescale increases the possibility that larger-scale, nonturbulent sub-mesoscale motions, which may not be related to the local mean wind speed or temperature gradient, will be included in the calculated flux, especially in stable conditions (e.g., Smedman, 1988; Vickers and Mahrt, 2006; Mahrt, 2009). $C_{H}$ increases with increasing perturbation timescale because of the systematic flux loss when using too small a value for $\tau$ to calculate the heat flux. For example, if there is significant heat flux associated with turbulent transport on timescales of $300 \mathrm{~s}$ and the flux is calculated using $\tau$ equal to $100 \mathrm{~s}$, then the computed flux will be an underestimation for the given wind speed and temperature gradient.

An alternative approach for estimating the sub-canopy $C_{H}$ is to compute individual $30 \mathrm{~min}$ average estimates, equal to the heat flux divided by the product of the mean wind speed and the temperature difference (Eq. 3). The frequency distribution of such estimates is shown in Fig. 8. This approach to estimate $C_{H}$ yields a mean value of $1.1 \times 10^{-3}$ with a standard deviation of $2.05 \times 10^{-3}$. When computing individual 30 min average estimates of $C_{H}$, we exclude cases where the product of the absolute value of the 30 min mean wind speed and the temperature difference is less than $0.1 \mathrm{Kms}^{-1}$. This is necessary to avoid dividing by a very small number or zero when solving Eq. (3) for $C_{H}$ for individual $30 \mathrm{~min}$ periods. About $15 \%$ of the $C_{H}$ estimates are less than zero, indicating counter-gradient heat transfer (Denmead and Bradley, 1985) or, more likely, very small mean fluxes and large random flux sampling errors. The negative values of $C_{H}$ tend to be associated with stronger wind speed periods. Some of the variation in the sub-canopy $C_{H}$ (Fig. 8) appears to be due to variation in stability (not shown). However, we avoid plotting $C_{H}$ as a function of $z / L$, where $L$ is the Obukhov length scale, to eliminate the problems associated with selfcorrelation, where the same quantity (in this case the heat flux) is contained within two different variables $\left(C_{H}\right.$ and $z / L$ ) being compared to each other (Hicks, 1978; Klipp and Mahrt, 2004; Baas et al., 2006). We also avoid comparing $C_{H}$ to a bulk Richardson number because both contain the temperature gradient. $C_{H}$ is positively related to the vertical velocity variance; however, models do not have information on the vertical velocity variance, so developing relationships based on the velocity variance may not be useful to parameterize the heat flux.

\subsection{Flux-gradient relationship above the canopy}

Above the canopy, $C_{H}$ is computed using a temperature difference equal to the brightness radiative canopy temperature minus the $38 \mathrm{~m}$ air temperature (top panels of Figs. 7 and
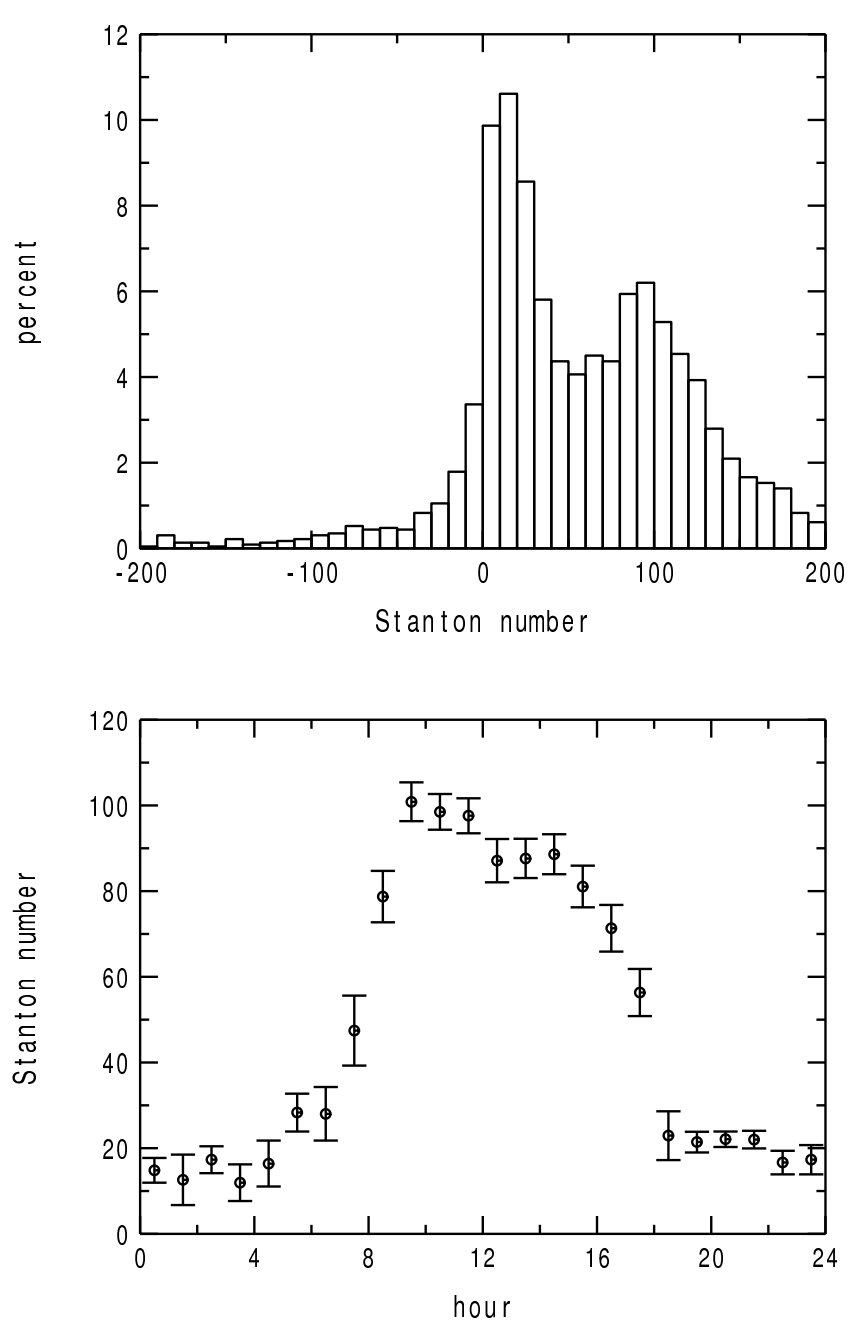

Figure 10. The frequency distribution (top panel) and the diurnal cycle (bottom) of the above-canopy Stanton number multiplied by 1000. Error bars denote \pm 1 standard error.

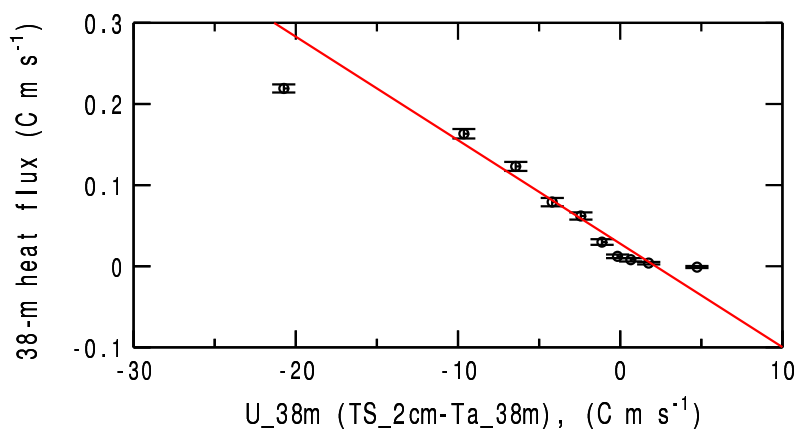

Figure 11. The kinematic heat flux as a function of the product of the mean wind speed and the temperature difference using the single-source approach (see text). The slope of the linear regression line (red) is estimate of the Stanton number: $-12.8 \pm 27.9 \times 10^{-3}$. Each of the ten class averages contains an equal number (282) of 30 min samples. Error bars denote \pm 1 standard error. 
9). With such a temperature gradient defined, our estimate of $C_{H}$ above the canopy using the regression slope method is $73.5 \pm 1.3 \times 10^{-3}$ (Fig. 9, top panel). This is about 65 times larger than the estimate found for the sub-canopy of $1.1 \pm 0.04 \times 10^{-3}$. The linear regression explains $77 \%$ of the variance of the heat flux at $38 \mathrm{~m}$ compared to $32 \%$ of the variance at $4 \mathrm{~m}$.

Stability appears to have a significant influence on the $38 \mathrm{~m} C_{H}$, leading to a bi-modal frequency distribution (Fig. 10). Stability is more important above the canopy compared to in the sub-canopy in part because the daytime heat fluxes at $38 \mathrm{~m}$ are typically 100 times larger than those in the sub-canopy. The mean daytime above-canopy $C_{H}$ is $84.2 \pm 55.6 \times 10^{-3}$, while the mean nighttime above-canopy $C_{H}$ is $15.3 \pm 26.4 \times 10^{-3}$. Again, direct comparisons between $C_{H}$ and $z / L$ or the bulk Richardson number are avoided here to eliminate contamination of the relationships by selfcorrelation.

One possibility to explain the larger $C_{H}$ above the canopy is that the larger surface area associated with the large leaf area density leads to more efficient transfer of heat for a given mean temperature difference and wind speed, and thus a larger exchange coefficient. Another possibility is simply that the turbulence is much stronger above the canopy; however, this difference in turbulence strength should be at least partially accounted for in the bulk formula where $U$ is related to the shear generation of turbulence and the temperature difference is related to the buoyancy generation of turbulence. While the larger normalized turbulence intensity above the canopy (0.32) compared to that in the sub-canopy (0.19) could account for some of the difference in $C_{H}$, it seems unlikely it could account for all of it. We would like to add that all three observational levels are likely located within the roughness sublayer, as it can extend from the surface to 3 to 5 times the canopy height (Garratt , 1980; Raupach and Thom , 1981; Thomas et al., 2006). If the $38 \mathrm{~m}$ measurements are indeed in the roughness sublayer, then the turbulence and the fluxes may be heterogeneous in the horizontal and much larger (or smaller) than predicted by standard flux gradient relationships.

\subsection{Single-source flux-gradient}

Here we estimate $C_{H}$ at $38 \mathrm{~m}$ using a single-source approach where the temperature difference used to estimate the heat flux at $38 \mathrm{~m}$ a.g.l. is computed as the $2 \mathrm{~cm}$ soil temperature minus the potential temperature of the air at $38 \mathrm{~m}$ a.g.l. That is, the single-source approach ignores the presence of the canopy.

Using the single-source approach, $C_{H}$ is negative $\left(-12.8 \pm 27.9 \times 10^{-3}\right)$, suggesting that the heat fluxes are counter-gradient (Fig. 11). That is, the single-source approach fails because it makes the heat flux appear to be counter-gradient when in fact the heat flux is aligned with the local temperature gradient in both the sub-canopy and abovecanopy layers when the two layers are considered separately.

Our presentation here of $C_{H}$ for the single-source approach is only an exercise for demonstration purposes. We are not aware which models, if any, may still be employing a single-source approach for grid points with tall forest canopies.

\section{Summary and conclusions}

The daytime sub-canopy heat flux is downward because the strong radiational warming takes place in the high leaf area density of the canopy layer, not at the ground. Conversely, the nighttime sub-canopy heat flux is upward because the strong radiational cooling takes place in the canopy layer, not at the ground.

The sub-canopy fluxes and vertical velocity variance exhibit greater peak timescales and broader spectral peaks compared to the those above the canopy. This likely indicates a variety of turbulence generation mechanisms in the subcanopy compared to above the canopy, where a single mechanism (buoyancy) dominates.

A peculiar double-peak structure with timescales of $0.8 \mathrm{~s}$ and $51.2 \mathrm{~s}$ is typically observed in the sub-canopy vertical velocity spectra during the day but almost never at night. The very small eddies responsible for the peak at $0.8 \mathrm{~s}$ may be associated with tree stem wake. A double-peak structure is also observed in the daytime sub-canopy heat flux co-spectra, where a local minimum in the negative heat flux is found for the range of timescales from 6.4 to $25.6 \mathrm{~s}$. We argue that the double peaks in the heat flux are the result of the canceling contribution of opposing heat fluxes creating a gap in an otherwise continuous co-spectra,

The daytime momentum flux co-spectra at $16 \mathrm{~m}$ and in the sub-canopy are characterized by a relatively large crosswind component, likely due to the extremely light and variable winds, such that the definition of a mean wind direction, and subsequent partitioning of the momentum flux into along- and cross-wind components, has little physical meaning. Positive values of both momentum flux components in the sub-canopy denote an upward transfer of momentum, consistent with the observed decrease in the mean wind speed with height between the sub-canopy and canopy layers.

The closed canopy appears to inhibit horizontal motions more than vertical motions, leading to large values of the velocity aspect ratio and the normalized turbulence intensity in the canopy. The suppression of the horizontal wind component compared to the vertical component was qualitatively confirmed by visualizing the flow using releases of buoyantly neutral fog. The sub-canopy motions are more anisotropic compared to those above the canopy, consistent with enhanced suppression of vertical velocity perturbations closer to the ground. 
The slope derived from linear regression of the sub-canopy heat flux as a function of the product of the mean wind speed and the temperature difference yields an estimate for the subcanopy Stanton number (exchange coefficient for heat) of $1.1 \pm 0.04 \times 10^{-3}$ (90\% confidence interval for the slope) The intercept of the regression is zero, indicating that, on average and despite the large scatter, the sub-canopy heat fluxes in the mean are aligned with the local temperature gradient. An alternative approach for estimating $C_{H}$, where we compute individual $30 \mathrm{~min}$ average estimates and average them, yields an estimate for the mean sub-canopy Stanton number of $1.1 \times 10^{-3}$ with a standard deviation of $2.05 \times 10^{-3}$.

The exchange coefficient for heat above the canopy is $73.5 \pm 1.3 \times 10^{-3}$, or about 65 times larger than the estimate found for the sub-canopy. Stability appears to have an important influence. The mean daytime (unstable) above-canopy $C_{H}$ is $84.2 \pm 55.6 \times 10^{-3}$, while the mean nighttime (stable) above-canopy $C_{H}$ is $15.3 \pm 26.4 \times 10^{-3}$. A likely explanation for the larger $C_{H}$ above the canopy compared to in the subcanopy is that the higher leaf area density in the canopy leads to more efficient transfer of heat compared to the ground surface for a given wind speed and temperature difference, and thus a larger exchange coefficient. Much stronger turbulence at $38 \mathrm{~m}$ may also be a factor, although this effect is typically assumed to be accounted for in the bulk formula.

A single-source approach for the $38 \mathrm{~m}$ heat flux fails because it neglects the presence of the canopy. Such failure is likely dependent on the canopy closure and vertical structure. Neglecting the canopy at our site makes the heat flux appear to be counter-gradient when in fact it is aligned with the local temperature gradient when the two layers are considered separately. This indicates that surface flux models that do not explicitly resolve the canopy and sub-canopy layers may compute erroneous heat fluxes.

Acknowledgements. This research was supported by the US Department of Energy (DOE), Office of Science (BER), contract DE-FG02-06ER64318 and the National Science Foundation (NSF), Physical and Dynamic Meteorology, Career award AGS0955444. We gratefully acknowledge the contribution of Kent Davis in collecting the field observations.

Edited by: L. Zhang

\section{References}

Baas, P., Steeneveld, G. J., Van De Wiel, B. J. H., and Holtslag, A. A. M.: Exploring self-correlation in flux-gradient relationships for stably stratified conditions, J. Atmos. Sci., 63, 3045-3054, 2006.

Baldocchi, D. D. and Meyers, T. P.: A spectral and lag-correlation analysis of turbulence in a deciduous forest canopy, Bound.-Lay. Meteorol., 45, 31-58, 1988.
Brunet, Y., Finnigan, J., and Raupach, M. R.: A wind tunnel study of air flow in waving wheat: single-point velocity statistics, Bound.Lay. Meteorol., 70, 95-132, 1994.

Denmead, O. T. and Bradley, E. F.: Flux-gradient relationships in a forest canopy, in: The Forest-Atmosphere Interaction, edited by: Hutchison, B. A. and Hicks, B. B., D. Reidel Publ. Comp., Dordrecht, Boston, London, 421-442, 1985.

Dupont, S., Irvine, M. R., Bonnefond, J., Lamaud, E., and Brunet, Y.: Turbulent structures in a pine forest with a deep and sparse trunk space: stand and edge regions, Bound.-Lay. Meteorol., 143, 309-336, 2012.

Garratt, J.R.: Surface influence upon vertical profiles in the atmospheric near-surface layer, Quart. J. Roy. Meteorol Soc, 106, 803-819, doi:10.1002/qj.49710645011, 1980.

Goulden, M. L., Munger, J. W., Fan, S. M., Daube, B. C., and Wofsy, S. C.: Measurements of carbon sequestration by longterm eddy covariance: methods and a critical evaluation of accuracy, Glob. Change Biol., 2, 169-182, 1996.

Hicks, B. B.: Some limitations of dimensional analysis and power laws, Bound.-Lay. Meteorol., 14, 567-569, 1978.

Howell, J. and Mahrt, L.: Multiresolution flux decomposition, Bound.-Lay. Meteorol., 83, 117-137, 1997.

Klipp, C. L. and Mahrt, L.: Flux-gradient relationship, selfcorrelation and intermittency in the stable boundary layer, Q. J. Roy. Meteor. Soc., 130, 2087-2104, 2004.

Mahrt, L.: Characteristics of submeso winds in the stable boundary layer, Bound.-Lay. Meteorol., 130, 1-14, 2009.

Mahrt, L.: Computing turbulent fluxes near the surface: needed improvements, Agr. Forest Meteorol., 150, 501-509, 2010.

Mahrt, L. and Vickers, D.: Bulk formulation of the surface heat flux, Bound.-Lay. Meteorol., 110, 357-379, 2004.

Meyers, T. P. and Baldocchi, D. D.: The budgets of turbulent kinetic energy and Reynolds stress within and above a deciduous forest, Agr. Forest Meteorol., 53, 207-222, 1991.

Oleson, K. W., Lawrence, D. M., Bonan, G. B., Flanner, M. G., Kluzek, E., Lawrence, P. J., Levis, S., Swenson, S. C., Thornton, P. E., Dai, A., Decker, M., Dickinson, R., Feddema, J., Heald, C. L., Hoffman, F., Lamarque, J., Mahowald, N., Niu, G., Qian, T., Randerson, J., Running, S., Sakaguchi, K., Slater, A., Stockli, R., Wang, A., Yang, Z., Zeng, X., and Zeng, X.: Technical Description of version 4.0 of the Community Land Model (CLM). NCAR Technical Note $N C A R / T N-478+S T R$, National Center for Atmospheric Research, Boulder, CO, 257 pp., 2010.

Raupach, M. R. and Thom, A. S.: Turbulence in and above plant canopies, Ann. Rev. Fluid Mech., 13, 97-129, 1981.

Raupach, M. R.: Simplified expressions for vegetation roughness length and zero-plane displacement as functions of canopy height and area index, Bound.-Lay. Meteorol., 71, 211-216, 1994.

Raupach, M. R., Finnigan, J., and Brunet, Y.: Coherent eddies and turbulence in vegetation canopies: the mixing-layer analogy, Bound.-Lay. Meteorol., 78, 351-382, 1996.

Smedman, A. S.: Observations of multi-level turbulence structure in a very stable atmospheric boundary layer, Bound.-Lay. Meteorol., 44, 231-253, 1988.

Staebler, R. M. and Fitzjarrald, D. R.: Measuring canopy structure and the kinematics of subcanopy flow in two forests, J. Appl. Meteorol., 44, 1161-1179, 2005.

Stull, R. B.: An Introduction to Boundary Layer Meteorology, Kluwer Academic Publishers, Boston, 666 pp., 1990. 
Thomas, C. K.: Variability of subcanopy flow, temperature, and horizontal advection in moderately complex terrain, Bound.-Lay. Meteorol., 139, 61-81, 2011.

Thomas, C.K., Mayer, J.C., Meixner, F.X., and Foken, T.; Analysis of low-frequency turbulence above tall vegetation using a Doppler sodar, Bound.-Lay. Meteorol., 119, 563-587, doi:10.1007/s10546-005-9038-0, 2006.

Thomas, C. K., Martin, J. G., Goeckede, M., Siqueira, M. B., Foken, T., Law, B. E., Loescher, H. W., and Katul, G.: Estimating daytime subcanopy respiration from conditional sampling methods applied to multi-scalar high frequency turbulence time series, Agr. Forest Meteorol., 148, 1210-1229, 2008.

Thomas, C. K., Martin, J. G., Law, B. E., and Davis, K. J.: Toward biologically meaningful net carbon exchange estimates for tall, dense canopies: multi-level eddy covariance observations and canopy coupling regimes in a mature Douglasfir forest in Oregon, Agr. Forest Meteorol., 173, 14-27, doi:10.1016/j.agrformet.2013.01.001, 2013.
Vickers, D. and Mahrt, L.: The cospectral gap and turbulent flux calculations, J. Atmos. Ocean. Tech., 20, 660-672, 2003.

Vickers, D. and Mahrt, L.: A solution for flux contamination by mesoscale motions with very weak turbulence, Bound.-Lay. Meteorol., 118, 431-447, 2006.

Vickers, D. and Thomas, C. K.: Some aspects of the turbulence kinetic energy and fluxes above and beneath a tall open pine forest canopy, Agr. Forest Meteorol., 181, 143-151, 2013.

Vickers, D., Thomas, C. K., and Law, B.: Random and systematic $\mathrm{CO} 2$ flux sampling errors for tower measurements over forests in the convective boundary layer, Agr. Forest Meteorol., 149, 7383, 2009.

Vickers, D., Irvine, J., Martin, J. G., and Law, B. E.: Nocturnal subcanopy flow regimes and missing carbon dioxide, Agr. Forest Meteorol., 152, 101-108, 2012. 